\title{
Precentral cerebellar vein in cystic astrocytomas of the vermis
}

\author{
LEO N. HOPKINS AND LOUIS BAKAY ${ }^{1}$ \\ From the State University of New York at Buffalo Medical School, \\ Department of Neurosurgery, Buffalo, New York, U.S.A.
}

SYNOPSIS A peculiar abnormality of the precentral cerebellar vein seen only in cystic astrocytoma of the vermis is reported. Implications of this angiographic finding are discussed. The importance of posterior fossa angiography (especially venography) in the diagnosis of posterior fossa mass lesions is emphasized.

Until recently, angiography has been of little help in the diagnosis of mass lesions of the posterior fossa. Advances in neuroradiology have now made posterior fossa angiography the procedure of choice in many cases. The anatomy and radiography of the veins of the posterior fossa have been detailed by Huang and Wolf $(1965,1966)$. Reports of angiographic (especially venographic) features of posterior fossa lesions now abound in the literature (Huang et al., 1968; Mani et al., 1968; Huang and Wolf, 1969, 1970; Takahashi, 1974).

1 Address for correspondence: Dr Louis Bakay, E. J. Meyer Memorial Hospital, 462 Grider Street, Buffalo, New York 14215, U.S.A. (Accepted 25 April 1975.)
Interpretation of venograms of the posterior fossa is difficult because of the variability of the venous anatomy. However, one of the most reliable venous structures is the precentral cerebellar vein (Huang and Wolf, 1966). In vertebral angiograms of good quality it is almost always visualized and its position is relatively constant in normal circumstances. It originates within the precentral cerebellar fissure, bounded by the central lobule of the cerebellum above and the lingula and anterior medullary vellum below (Fig. 1). The vein is either paired or consists of several small parallel veins which run forward within the precentral cerebellar fissure, parallel to the roof of the fourth ventricle. A common trunk

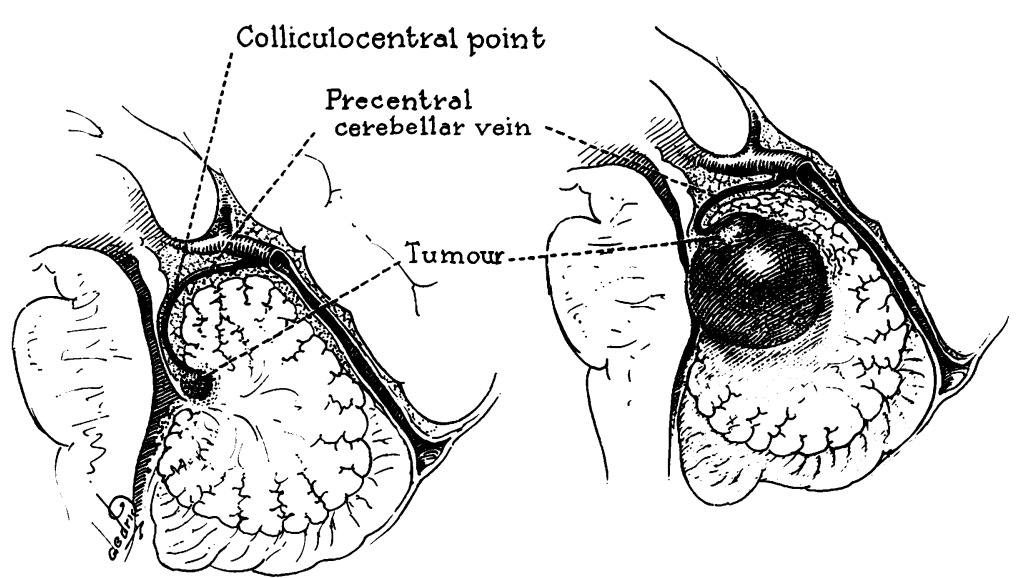

FIG. 1 Artist's conception of the normal anatomy of the precentral cerebellar vein and the origin of the tumour beneath the vein (left). The right side shows the resulting deformity of the vein as the tumour enlarges in size. Compare this deformity with that seen_in_the angiogram in Fig. 2. 


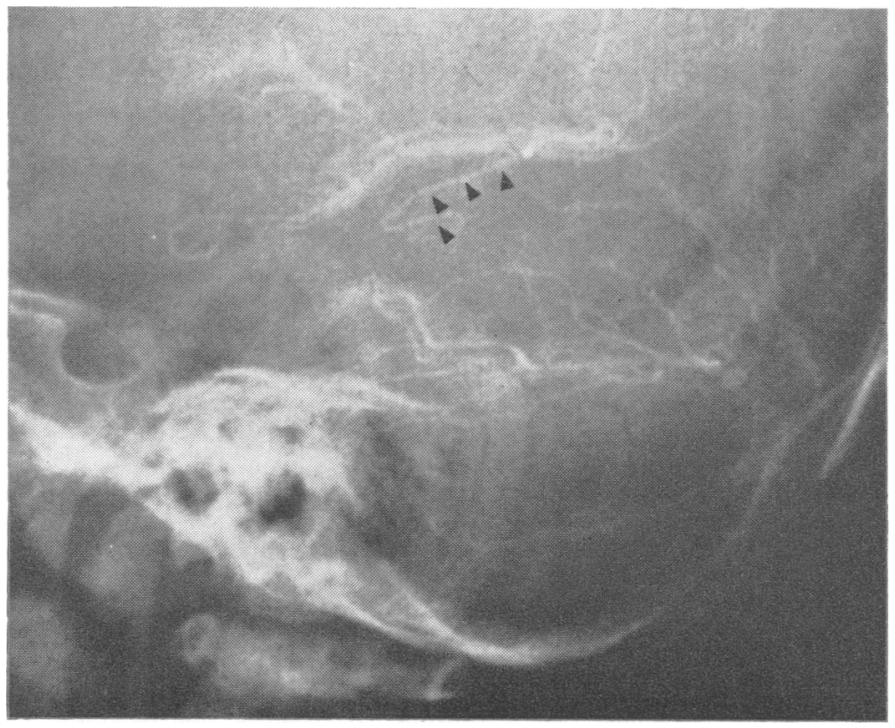

FIG. 2 Preoperative posterior fossa venogram in one of the patients with cystic astrocytoma of the cerebellar vermis. Note the characteristic deformity of the precentral cerebellar vein (dark arrows).

is eventually formed within the fissure, which passes out of the precentral cerebellar fissure and curves upward into the quadrigeminal cistern. It enters the cistern at a point between the inferior colliculi below and the central lobule above. This point is the so-called colliculocentral point (Fig. 1). The vein then curves gently upward to its point of junction with the vein of Galen. The precentral cerebellar vein is usually clearly visible on lateral posterior fossa venograms. On anteroposterior (A-P) views, however, it is often obscured by superimposed vermian veins (Huang and Wolf, 1966). The fact that the precentral cerebellar vein in its initial portion often exists as several small parallel veins also makes identification on A-P views very difficult.

In the past year, we have seen three very similar tumours in children which all caused a rather characteristic angiographic deformity of the precentral cerebellar vein. In each case the juvenile astrocytoma presented as a large cyst with mural nodule occupying the cerebellar vermis superiorly. The mural nodule lay in the area of the roof of the fourth ventricle superiorly, displacing the ventricle forward. The cyst occupied the superior vermis. In all three of our cases the precentral cerebellar vein was displaced forward and upward; the terminal portion showed a peculiar hook-like deformity (Fig. 2).
The correlation between the characteristic displacement of the precentral cerebellar vein angiographically and the pathology seen at surgery has two implications. First, in tumours of this type a reasonable estimate of the pathology can be made from the characteristic deformity of the precentral cerebellar vein. Second, the striking deformity of the precentral cerebellar vein suggests that the origin of these tumours must have been in the region of the anterior medullary vellum or lingula of the cerebellum. Figure 1 depicts the anatomy of the precentral cerebellar vein within the precentral fissure and quadrigeminal cistern. Its intimate relationship with the lingula and the anterior medullary vellum below and the remainder of the vermis above is obvious. Our concept of the origin of these three tumours in the lingula cerebelli and the resulting deformity of the precentral cerebellar vein is shown in the same diagram.

The forward and upward displacement in combination with the hook-like deformity of the distal end of the vein implies that the tumour originates beneath the distal portion of the precentral cerebellar vein-that is, in the lingula or anterior medullary vellum. As the tumour enlarges, the overall forward and upward displacement occurs.

Most tumours arising in the superior vermis 
of the cerebellum result in forward displacement of the precentral cerebellar vein as a whole (Rosa and Viale, 1970; Peeters, 1973; Takahashi et al., 1973; Takahashi, 1974). Tumours occurring in the lumen of the fourth ventricle and anterior to it - that is, brain stem tumours and extra-axial tumours ventral to the brain-stemcause backward or posterior displacement of the precentral cerebellar vein (Huang and Wolf, 1969, 1970; Takahashi, 1974). The smooth forward displacement with terminal hook-like deformity seen in these three cases has not been observed in any but cystic astrocytomas of the superior cerebellar vermis. We are confident that this will represent a helpful angiographic sign in the diagnosis of cerebellar vermis astrocytomas.

This study was supported by the Buswell Foundation.

\section{REFERENCES}

Gabrielsen, T. O., and Amundsen, P. (1969). Pontine arteries in vertebral angiography. American Journal of Roentgenology, Radium Therapy and Nuclear Medicine, 106, 296-302.

Huang, Y. P., and Wolf, B. S. (1965). Veins of posterior fossa-superior or galenic draining group. American Journal of Roentgenology, Radium Therapy and Nuclear Medicine, 95, 808-821.
Huang, Y. P., and Wolf, B. S. (1966). Precentral cerebellar vein in angiography. Acta Radiologica, Diagnosis, 5, 250262.

Huang, Y. P., and Wolf, B. S. (1969). Angiographic features of fourth ventricle tumors with special reference to posterior inferior cerebellar artery. American Journal of Roentgenology, Radium Therapy and Nuclear Medicine, 107, 543-564.

Huang, Y. P., and Wolf, B. S. (1970). Angiographic features of brain stem tumors and differential diagnosis from fourth ventricle tumors. American Journal of Roentgenology, Radium Therapy and Nuclear Medicine, 110, 1-30.

Huang, Y. P., Wolf, B. S., Antin, S. P., and Okudera, T. (1968). Veins of posterior fossa-anterior or petrosal draining group. American Journal of Roentgenology, Radium Therapy and Nuclear Medicine, 104, 36-56.

Mani, R. L., Newton, T. H., and Glickman, M. G. (1968). Superior cerebellar artery: anatomic-roentgenographic correlation. Radiology, 91, 1102-1108.

Peeters, F. L. (1973). The vertebral angiogram in patients with tumors in or near the midline. Neuroradiology, 5 , 53-58.

Rosa, M., and Viale, G. L. (1970). Diagnostic value of vertebral phlebogram. Neuroradiology, 1, 147-150.

Takahashi, M. (1974). Atlas of Vertebral Angiography. University Park Press: Baltimore.

Takahashi, M., Okudera, T., Tanaka, M., Kitamura, K., and Yonemasu, Y. (1973). Angiographic diagnosis of cerebellar medulloblastomas: evaluation of pre- and postoperative vertebral angiographies. American Journal of Roentgenology, Radium Therapy and Nuclear Medicine, 118, 622-632.

Wolf, B. S., Huang, Y. P., and Newman, C. M. (1963). Lateral anastomotic mesencephalic vein and other variations in drainage of basal cerebral vein. American Journal of Roentgenology, Radium Therapy and Nuclear Medicine, 89, 411-422. 